\title{
Comprehensive phenotyping in multiple sclerosis: Discovery based proteomics and the current understanding of putative biomarkers
}

\author{
Kevin C. O'Connor ${ }^{\mathrm{a}, *}$, Sushmita Mimi Roy ${ }^{\mathrm{b}}$, Christopher H. Becker ${ }^{\mathrm{b}}$, David A. Hafler ${ }^{\mathrm{a}}$ and \\ Aaron B. Kantor ${ }^{b}$ \\ ${ }^{a}$ Department of Neurology and the Center for Neurologic Disease, Brigham and Women's Hospital, Laboratory of \\ Molecular Immunology, Department of Neurology, Harvard Medical School 77 Avenue Louis Pasteur, Boston, MA \\ 02115, USA \\ ${ }^{\mathrm{b}}$ Biomarker Discovery Sciences, PDD, 1505 O’Brien Drive, Menlo Park, CA 94025, USA
}

\begin{abstract}
Currently, there is no single test for multiple sclerosis (MS). Diagnosis is confirmed through clinical evaluation, abnormalities revealed by magnetic resonance imaging (MRI), and analysis of cerebrospinal fluid (CSF) chemistry. The early and accurate diagnosis of the disease, monitoring of progression, and gauging of therapeutic intervention are important but elusive elements of patient care. Moreover, a deeper understanding of the disease pathology is needed, including discovery of accurate biomarkers for MS. Herein we review putative biomarkers of MS relating to neurodegeneration and contributions to neuropathology, with particular focus on autoimmunity. In addition, novel assessments of biomarkers not driven by hypotheses are discussed, featuring our application of advanced proteomics and metabolomics for comprehensive phenotyping of CSF and blood. This strategy allows comparison of component expression levels in CSF and serum between MS and control groups. Examination of these preliminary data suggests that several CSF proteins in MS are differentially expressed, and thus, represent putative biomarkers deserving of further evaluation.
\end{abstract}

\section{Introduction}

This article will briefly review our current knowledge of biomarkers in multiple sclerosis (MS), with particular focus on neuroimmunology, and especially antibodies. It is not intended as an exhaustive review, as such work has recently been completed in several excellent and thorough publications [1-3]. The later part of the manuscript will focus on the appraisal of groundwork biomarker discovery in MS using our own novel strategy, which allows detection, identification, and quantification of a large number of putative biomarkers in

${ }^{*}$ Corresponding author: Kevin C. O'Connor, Ph.D., Harvard Medical School, 77 Avenue Louis Pasteur NRB641, Boston, MA 02115, USA. Tel:: +1 617525 5348; Fax: +1 617525 5333; E-mail: koconnor@rics.bwh.harvard.edu.
MS, leading to a "molecular signature" of proteomic patterns free of pre facto bias.

\section{Multiple sclerosis}

MS, the most common autoimmune disease involving the nervous system (CNS), is a chronic illness affecting CNS pathways and leading to progressive neurological dysfunction [4,5]. The disease affects twice as many women as it does men. Approximately 400,000 Americans acknowledge having MS, and every week about 200 people are diagnosed. Worldwide, MS may affect 2.5 million individuals. The high economic burden is reflected in the total estimated annual cost for all people with MS in the United States: more than $\$ 9$ billion dollars [6]. Both genetic and environmental factors contribute to MS susceptibility. High-risk re- 
gions include the northern United States and the Scandinavian countries, while Japan and Africa are areas of lower risk. Individuals that migrate from high-risk to low-risk regions carry their native risk for contracting MS, suggesting that exposure to an environmental factor contributes to susceptibility. Genetic studies have shown that the risk of developing MS is elevated 10to 20-fold in first-degree relatives of individuals with MS and that the concordance rate among monozygotic twins is $30 \%-35 \%$, but only $2 \%-5 \%$ in dizygotic twins.

Clinically, the disease can be broadly divided into a relapsing-remitting form characterized by a series of exacerbations that result in varying degrees of disability from which the patient recovers, and two progressive forms (secondary progressive, SP and primary progressive, PP) in which the patient does not experience exacerbations, but instead experiences a gradual decline. Though a relapsing-remitting onset is observed in $85-90 \%$ of all patients, the course of the disease in about $40 \%$ of them ultimately becomes progressive [5]. Thus, the current prevalence of the subtypes is approximately $45 \%$ RR, $35 \%$ SP, and $20 \%$ PP. The pathological hallmark of MS is the demyelinated plaque found throughout the brain and spinal cord, with CNS perivascular infiltration of inflammatory cells, demyelination, astrogliosis, and axonal injury (reviewed in references [7-10]). Symptoms are believed to result primarily from axonal demyelination that inhibits or blocks conduction. Amelioration of symptoms has been attributed to both partial remyelination and resolution of inflammation.

It is widely accepted that MS is a complex syndrome and may not represent a single disease entity. Based on accumulating data from immunological studies of patients with MS and a wealth of animal model data, autoimmune dysregulation has been viewed as the major contributor to tissue damage (reviewed in references [9-11]. There is widespread support for the view that $\mathrm{MS}$ is an immune-mediated disease, based largely on: 1) the genetic linkage to immune-related molecules, primarily MHC class II; 2) the presence of CNS inflammatory infiltrates including CD4 ${ }^{+} \mathrm{T}$ cells, $\mathrm{CD}^{+} \mathrm{T}$ cells, B cells, plasma cells, macrophages; 3 ) magnetic resonance imaging (MRI) indicative of the loss of integrity of the blood - brain barrier (BBB); 4) the response to immune-modulating therapies; 5) similarities to the animal model of experimental autoimmune encephalomyelitis (EAE); and 6) the presence of oligoclonal bands and elevated immunoglobulin levels in the cerebrospinal fluid (CSF).

In accordance with this evidence, a widely accepted model of MS immunopathology [12] suggests that ac- tivated autoreactive $\mathrm{T}$ cells recognize myelin antigens in the periphery, cross the CNS endothelium, leading to a cascade of events that culminates in white matter inflammation and tissue destruction. Inside the CNS, release of local cytokines, chemokines, and matrix metallo-proteins support the recruitment of subsequent waves of infiltrating effector cells (including monocytes) and B cells. Mechanisms of myelin destruction and axonal damage are likely to be multiple and include direct effects of pro-inflammatory cytokines, complement-fixing antibodies, antigen-specific and non-specific cytotoxicity, and apoptosis.

While it is broadly appreciated that a sizeable percentage of MS cases involve immune-mediated pathogenesis, neurodegenerative processes not necessarily associated with inflammation appear to be principal in others. Theories that overlook inflammation as a primary event hold that MS is at least partially a genetically determined disorder characterized by metabolic neurodegeneration. Studies of the mechanisms of axonal damage and neurodegeneration in MS are still in their infancy, though recognition of axonal damage as a prominent pathological feature in MS lesions is increasing (reviewed in references [7,13-15]).

It is likely that both neurodegeneration and inflammation contribute to various extents, both among and within individuals with MS. In a seminal work underscoring the significance of both inflammatory and non-inflammatory mechanisms in MS pathology, Lassman and colleagues [16] catalogued lesions and demonstrated the existence of four fundamentally different patterns of demyelination in humans. While two patterns (I and II) showed close similarities to T-cellmediated or T-cell plus antibody-mediated autoimmune encephalomyelitis, the other patterns (III and IV) were highly suggestive of a primary oligodendrocyte dystrophy, reminiscent of demyelination induced by a virus or toxin rather than one involving the immune system.

\section{Biomarkers}

Biomarkers are anatomic, physiologic, biochemical, or molecular parameters associated with the presence and severity of specific disease states. Biomarkers are measurable by a variety of methods including physical examination, laboratory assays, and medical imaging. The hallmark of a useful biomarker is that the state of health or disease of an individual can be accurately characterized by appraisal of the marker [17]. Thus, biomarkers can indicate either nor- 
mal biological/pathogenic processes or pharmacological responses to a therapeutic intervention. Simple examples include CD4+ T-cell count in HIV-infected patients and HDL/LDL ratios for patients with hypercholesterolemia. Some biomarkers are not specific; prostate-specific antigen (PSA) may be increased not only in men who have prostate cancer, but also in those with benign prostatic hyperplasia or a prostate infection. More pertinent to the present report, white matter abnormalities, which can be detected by MRI, serve as biomarkers for MS.

\section{Biomarkers in MS inflammation}

The current inventory of prospective MS biomarkers by and large includes those that reflect the changeable inflammatory processes in the majority of cases. Many such biomarkers are found in the periphery, namely in the serum. However, analysis of CSF can add greater pathologic specificity, as it is most closely associated with the disease process. Thus, considerable efforts have been made to identify prognostic and diagnostic markers in the CSF from patients with MS.

The biomarkers reflecting the role of inflammation include cytokines, chemokines, adhesion molecules, and each of their respective receptors, complement, and antibodies. Specifically, the pro-inflammatory cytokines associated with T cells; interferon (IFN)- $\gamma$, tumor necrosis factor (TGF)- $\alpha$ and IL-12 may be expressed at elevated levels. Correspondingly, the antiinflammatory cytokines such as IL-4, IL-10, and TGF$\beta$ may be overexpressed during periods of remission. In each of these cases, more evidence is required.

Breakdown of the blood-brain barrier (BBB) is necessary in some cases before immune effector cells can extravasate into the CNS. In other cases T cells can extravasate through an intact BBB. Disruption of the BBB involves proteolytic activity of enzymes that degrade the extracellular matrix (ECM), chief among which are the matrix metalloproteinases (MMPs). Accordingly, elevated expression levels of MMP family members in patients with MS have been reported.

In addition to larger proteins, small-molecule biomarkers have also been investigated. For instance, it is appreciated that nitric oxide (NO) is formed in inflammatory disorders. Consequently, levels of NO, its oxidation products (NOx), and the iNOS enzyme are altered. Several studies have examined particular parts of this response. CSF nitrite levels appear to be correlated with disease exacerbation and concurrent inflammation in the CNS of patients with MS [18].

It is well-accepted that autoantibodies toward insulin and DNA are established biomarkers in the autoimmune diseases type 1 diabetes (T1D) and systemic lupus erythematosus, respectively. However, these autoantibodies have yet to be implicated directly in disease pathogenesis. In contrast, myasthenia gravis autoantibodies directed toward components of the neuromuscular junction are both diagnostic biomarkers and participants in disease pathology [19]. Neuromyelitis optica, (NMO) also termed Devic's syndrome, is an inflammatory demyelinating disease that affects the optic nerves and spinal cord, but unlike MS, does not affect the brain. Early in its course, Devic's is difficult to distinguish from MS, yet the distinction remains important, as treatment and prognosis of these two diseases are dissimilar. The presence of IgG serves as a specific serologic biomarker for early diagnosis of NMO and distinction from MS [20]. The NMO serum IgG reveals a characteristic pattern upon immunohistochemical staining of mouse CNS tissue. Similarly, Vincent and colleagues [21] found that the serum Ig of patients with MS recognized antigens on the surface of CNSderived cells, whereas serum Ig from controls did not. In this case, as with NMO, the appropriate next step in biomarker refinement would be identification of the target(s).

As autoantibodies have been shown to be useful markers in some autoimmune diseases, these molecules have also been examined for their suitability as biomarkers in MS. Other approaches have focused on individual candidate antigens. Consequently, there is an abundance of reports concerning autoantibodies in MS. Although much of this work has focused on the role of autoantibodies in the immunopathogenesis of MS, most are still only putative biomarkers, as a direct role in pathology has not been reported. That said, no study has clearly demonstrated that autoantibodies in MS are either reliable biomarkers or contributors to the disease pathology. However, when considered collectively, the reports on EAE and MS certainly indicate that suspicion of such a role for autoantibodies is likely well-founded. The evidence for a consequential humoral response within patients with MS includes the presence of CSF oligoclonal bands [22] and intrathecal immunoglobulin synthesis [23], both hallmarks of MS and diagnostic biomarkers used in conjunction with clinical presentation and MRI. This combination of several biomarkers is necessary, because CSF oligoclonal bands and evidence of intrathecal im- 
munoglobulin synthesis are found in conditions other than MS, such as subacute sclerosising panencephalitis (SSPE). Again, this underscores the dictum that no single biomarker is likely to be conclusive, but rather an arrangement of at least several biomarkers, forming a profile or fingerprint, will be needed to provide valuable information.

The possibility that CSF Ig in patients with MS is generated as a response to myelin self-antigens has also been investigated in detail. Antibodies specific for MBP have been identified in the CSF [2426]. Autoantibodies reactive with myelin-associated glycoprotein (MAG) [27], the enzyme transaldoase (TAL) [28], MOG [29], and oligodendrocyte-specific protein (OSP) [30] have also been detected in the CSF of a subset of patients with MS. One series of reports [31] demonstrated that individuals with MS who have intrathecal synthesis of $\operatorname{IgM}$ were likely to progress from RRMS to a more severe progressive form. The presence of intrathecal IgM correlates with progression from initial stages to clinically definite MS [32]; higher EDSS scores [33] and a fraction of this $\operatorname{IgM}$ reactive with myelin lipids suggests a more aggressive course [34].

Serum anti-MBP antibodies have been reported [35, 36], though not in all studies [37-39]. As with MBP, reports are conflicting regarding the detection of antiMOG antibodies in MS serum [29,40]. There are reports detecting serum autoantibodies against recombinant TAL [28], and B cells secreting autoantibodies directed against PLP have been identified in the peripheral blood of patients with MS [41]. Autoantibodies to other putative MS antigens, such as MOBP, CNPase, $\alpha ß$-crystalline, and $\mathrm{S} 100 \mathrm{~B}$ have not been analyzed in sera [42].

Autoantibodies directed against non-myelin antigens have also been detected in the sera of some patients with MS. Anti-nuclear antibodies (ANA) [43], anticardiolipin antibodies [44], and antibodies against beta2-glycoprotein I [45] are detected in MS sera more frequently than in normal subjects. Recently, elevated levels of antibodies towards a large panel of organ- and non-organ-specific antigens were reported in patients with MS relative to controls [46]. Currently, no B-cell antigen specific for MS or identifying a subtype of MS has been established as a true biomarker for MS.

Our own studies have indicated that the antibodies to chief candidate myelin antigens, MBP and MOG, in the serum and CSF of patients with MS, are not readily detectable $[47,48]$ and therefore fail as useful biomarkers. Our work has shown that patients with acute dis- seminated encephalomyelitis (ADEM), an induced encephalomyelitis, have these autoantibodies. Moreover, reports concerning their detection in ADEM are consistent $[47,49]$, which cannot be said of those concerning MS. Our view is that the inconsistency in the reported data of autoantibodies in MS is caused by false positives generated by acute-phase reactants that result in "sticky" antibodies. Alternatively, the difficulty in consistently detecting autoantibodies in the periphery of patients with MS is that they may be produced locally within the CNS at the site of injury. The evidence for a significant humoral response within the CNS of patients with MS includes the presence of plasma cells and IgG in CNS lesions [16,50]. That an antigen-driven response is at work is indicated in analysis of the immunoglobulin VH and VL domains from CNS samples, which reveals that these gene segments have accumulated replacement mutations in the complementary determining regions (CDRs) [51] and a skewed variable region repertoire [51,52]. These findings strongly suggest an antigen-driven response, rather than polyclonal $\mathrm{B}$ cell activation [53-55]. Moreover, autoantibodies to MOG were found to be bound to disintegrating myelin segments in MS lesions [56] and in IgG eluates from MS lesions [48], supporting the contention that myelin autoantibodies may mediate CNS tissue damage. IgG isolated from CNS tissue binds to MBP in solid-phase assays [57]. Collectively, these results support the hypothesis that pathologically significant antibodies are produced within the brain and may constitute specific biomarkers. Measurement of these potentially useful biomarkers is clearly limited by our current technology. Application of novel non-invasive technology or more sensitive assays for measurement in the periphery may provide access to these prospective biomarkers.

\section{Biomarkers in MS neurodegeneration}

The neuropathology of MS largely concerns the CNS white matter, although lesions may sometimes be found in the gray matter as well. The key cellular components of the white matter are the myelin-coated axons responsible for conducting nerve impulses. The remaining fundamental CNS support cells are categorized as glial cells and include: oligodendrocytes, which provide and maintain the axonal myelin sheaths; astrocytes, which provide mechanical and metabolic support; and microglia, which perform phagocytosis, essentially becoming activated to clean up after cell damage or death. 
The gray matter is largely comprised of neurons that collect, integrate, and transmit information.

Certain traditional concepts regarding MS pathogenesis have recently come into question. For instance, it is now evident that the types of cells and molecules involved in MS are more diverse than previously thought. In particular, some CNS lesions can include either extensive oligodendrocyte apoptosis, microglial activation, axonal damage, or all of these features, while containing few or no lymphocytes [58]. While these observations do not preclude the role of inflammatory demyelination in MS pathogenesis, this form of CNS tissue damage may predate the inflammatory lesions. Thus, independent axonal pathology may contribute to the primary pathobiology of the disease.

Thus, there has been a renewed interest in the neurodegenerative mechanisms of MS. The most reported tissue injury is oligodendrocyte apoptosis and axonal degeneration and transection. Thus, irreversible neurologic disability in MS is related, in part, to axonal damage. All these pathological findings can be detected on MRI as atrophy and hypointense T1-weighted lesions, but pathogenic specificity is lacking.

Evaluation of glial cell responses in CSF and lesion tissue [59] revealed that $\mathrm{S} 100 \mathrm{~B}$ is a worthy marker for the relapsing phase of the disease and can be used along with ferritin to discriminate among the types of MS. Glial-fibrillary acidic protein (GFAP) correlated with disability scales. In another bona fide demonstration of the importance of combinations of biomarkers, the S100B: ferritin ratio discriminated patients with RR multiple sclerosis from SP, PP, or control patients and may therefore provide a marker for irreversible damage.

Markers of tissue destruction are useful for gauging neurodegenerative processes in MS. Isoprostanes, formed by free radical peroxidation, are emerging as a new class of sensitive, specific, and reliable markers of free radical damage and lipid peroxidation. CSF levels of isoprostane are elevated in MS patients [60]; however, in this context the elevation is related to degenerative rather than inflammatory phenomena [61]. Magnetic resonance spectroscopy can be used to detect levels of whole brain $\mathrm{N}$-acetylaspartate (NAA); the decreases seen in MS suggest progressive neuronal cell loss. Though whole-brain NAA measurement offers a quick, highly reproducible measure of disease progression, its use is dependent upon access to MRI instrumentation.

Some putative markers that deserve further investigation and validation include cytoskeleton structural proteins like actin, tubulin, L-neurofilaments and tau pro- tein, axon-specific antibodies, other neuronal or glial proteins like S-100, 14-3-3 and glial fibrillary acid protein, neuronal specific enolase, and nitric oxide and its metabolites (reviewed in reference [62]). However, none currently fulfils the criterion of applicability in clinical practice.

Transected or injured axons release several molecules from their proximal extremity into the intercellular space. Both the neurofilaments contained in the cytoplasm of neurons and the autoantibodies directed toward them have been viewed as potential biomarkers in MS and other neurological disorders [63]. Investigators have searched for correlations between levels of these markers and clinical or MRI measures with success, albeit limited.

\section{Biomarkers in MS: Proteomics, the next generation technology}

Despite tremendous efforts in MS research, the specific targets of the immune response, the precise mechanism for neuronal loss, and the events leading to disease etiology are not yet clear. Better biological markers that represent the biological activity of the disease process and response to therapy are needed. Although numerous putative MS-specific biomarkers, representing different mechanisms of pathogenesis and steps along the inflammatory cascade have been proposed., none has been fully validated. As one can deduce from the descriptions of biomarkers above, most of this work has been limited to investigation of one to several markers at a time. Although many advances have been made, we are still faced with exploring complex systems with studies designed to illuminate only one or a few of these multi-level biological problems. Comprehensive proteomic phenotyping fills the unmet need for rapid and methodical examination of molecules not only in the immune system but also from the targeted organs. To comprehend a disease as enigmatic and heterogeneous as MS, a high throughput technology capable of profiling multiple changes is needed.

We have taken a high-throughput multiplexed proteomics and metabolomics approach to biomarker identification, using both discovery-based and hypothesisbased strategies [64-66]. Biological samples are analyzed by mass spectrometry because of its high sensitivity, information content, and throughput. Our approach does not require any a priori assumptions about the specific differences between sample groups. Moreover, recently developed software and bioinformatics 
infrastructure enable the comparison of large numbers of samples in a single study. Comparison of multiple biological samples relies on the linearity of signal versus molecular concentration, reproducibility of sample processing, and overall spectral intensity normalization. Thousands of molecular components can be tracked from hundreds of samples with this approach [67-69]. Mass spectrometry measures the mass-to-charge $(\mathrm{m} / \mathrm{z})$ ratio of proteins, peptides, nucleic acids, carbohydrates, or metabolites. The measurements are carried out in the gas phase on ionized analytes. The instrument consists of an ion source, a mass analyzer that measures the mass-to-charge ratio $(\mathrm{m} / \mathrm{z})$ of the ionized analytes, and a detector that registers the number of ions at each $\mathrm{m} / \mathrm{z}$ value. Details of the various techniques, with particular focus on proteomics, have been comprehensively reviewed [70]. Electrospray ionization (ESI) is our preferred method for quantitative differential profiling. ESI is very stabile and enables direct on-line coupling of liquid chromatography to mass spectrometry by spraying the eluent into the mass spectrometer under high voltage and charging conditions. The microdroplets formed evaporate, leaving ionized analytes for mass analysis.

\section{Biomarkers in MS: Proteomics, results}

Our method for differential profiling of proteins and low molecular weight molecules from biological samples has three main technical components: 1) sample preparation, 2) liquid chromatography-mass spectrometry instrumentation, and 3) data processing informatics $[65,67,69]$. The overall separation and analysis scheme is shown in Fig. 1. Initial sample preparation, prior to chromatography, varies with the biological sample, such as serum, CSF, urine, or cells; whereas the instrumentation and software generally apply across sample types. The sample is divided into a fraction with high molecular weight (proteome) and another with low molecular weight (metabolome, including free-floating peptides), since these require different methods of preparation, mass spectrometry and identification. Here we focus on the proteome. Highabundance proteins, e.g., albumin and immunoglobulin, which account for about $90 \%$ of the serum proteins, are removed to enable better monitoring of lowerabundance proteins. The proteome is denatured, reduced, alkylated, and trypsin-digested to generate peptides, which are easier to resolve than large intact proteins. This approach enhances reproducibility and identification. The digested sample is then applied to on-line reverse-phase liquid chromatography, directly coupled to electrospray ionization mass spectrometry with time-of-flight (TOF) detection. TOF detection is ideal for distinguishing individual components in complex mixtures, since it provides high resolution and high mass accuracy, to an optimum of $2 \mathrm{mDa}$.

An important technical advance for the mass spectrometry platform was the development of software to pick peaks and make comparisons across a large number of different samples $[67,71,72]$. The data processing includes 1) removing and smoothing of baseline noise and spikes from the raw data, 2) dynamic thresholding to select peaks in different regions of the spectrum, 3) considering both the $\mathrm{m} / \mathrm{z}$ and chromatographic dimensions to eliminate chemical noise and identify peaks pertaining to true analyte molecular ions, 4) adjusting for small deviations in the elution time with dynamic time warping algorithms, and 5) comparing each file to a reference file to generate a single constant based on the median of the ratios of intensities for all components between the reference and test files and normalizing the complete data set. De-isotoped peaks at a certain frequency (e.g. one out of four samples in a two-group cross-sectional comparison) are retained in a merged peak list for statistical evaluation. Data on system performance, including reproducibility and linearity of the response, can be found in Wang et al. and Roy et al. [67,68]. One-dimensional chromatography with a hydrophobic reverse-phase column currently tracks more than 45,000 molecular components in the serum proteome. More molecular components can be monitored if the sample is separated using two orthogonal chromatography methods. As outlined in Fig. 1 on the right, we use strong cation exchange, with collection of up to eight fractions, followed by the same reverse-phase chromatography used in the onedimensional method. This enables tracking of about 30,000 molecular species [69].

Separating the differential profiling steps from peptide identification is an extremely useful strategy for improving throughput and reproducibility. Peptide identification is made using established tandem mass spectrometry approaches (MS/MS). Collisional activation for tandem mass spectrometry provides fragments (usually broken at the peptide bond) that can be assigned using a match to large public databases via the SEQUEST and MASCOT computer programs [73]. For new studies, links are first made to our library of identified peptides based on retention time and mass. Additional significant differences between groups (such as 


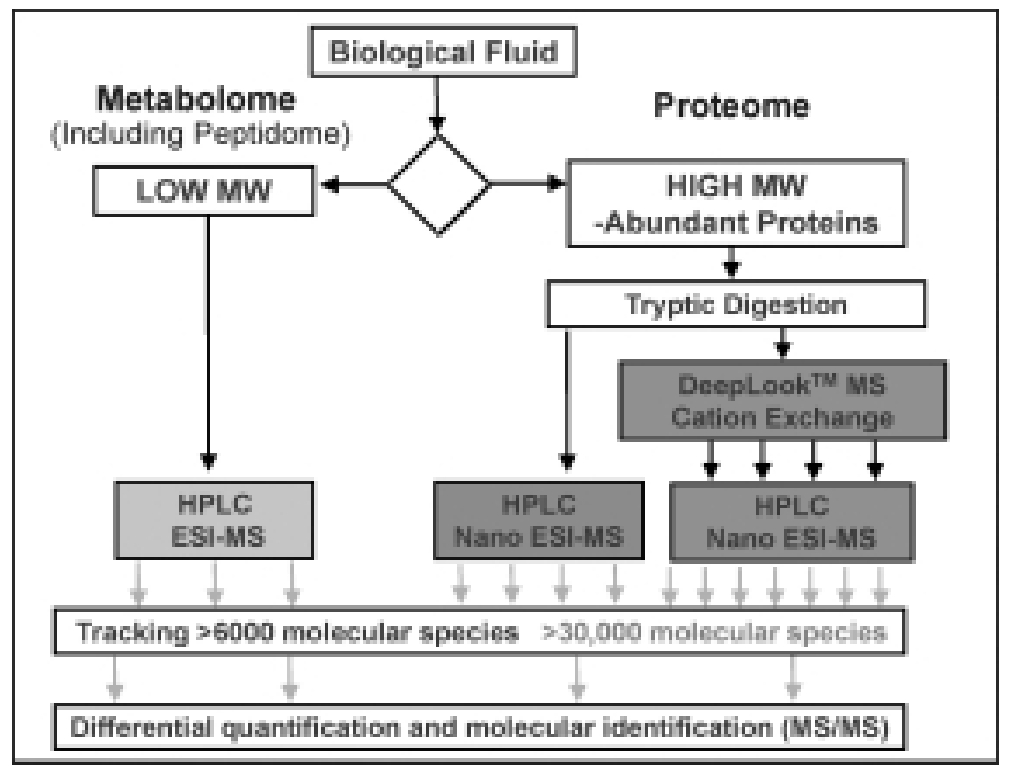

Fig. 1. Schematic diagram of the sample preparation and mass spectrometry analysis. Biological samples can be processed through the mass spectrometry procedures shown here. For serum, $>6,000$ molecular species can now be tracked with the metabolomic and proteomic one-dimensional approaches using current MassView ${ }^{\mathrm{TM}}$ software. The two-dimensional approach, with a strong cation exchange (SCX) separation before the reverse-phase HPLC, allows more than 30,000 molecular species to be tracked. Identification is done separately from the profiling. This enables higher throughput processing and allows identification to be focused on components with the greatest difference between the groups. Once a molecular ion is identified, the information is listed in the database for reference in future analysis.

cases of MS and healthy subjects) are considered based on p-values and magnitudes of differences and then identified with directed tandem mass spectrometry.

To address our first goal of serum profiling, we collected serum from 20 patients with relapsing-remitting MS and 20 normal controls. A diagnosis of MS was confirmed based on the criteria of McDonald [74]. Serum was fractioned into the metabolome and proteome and processed as described above with onedimensional chromatographic separation. We also extended the analysis to the examination of CSF. In MS, it is expected that CSF more closely reflects events in the CNS than does peripheral blood and hence is the preferred compartment for initiation of biomarker discovery efforts, despite being more difficult to obtain. Although most proteins $(\sim 80 \%)$ in the normal CSF originate from the blood, they are generally reduced 100to 1,000 -fold. Blood proteins passively diffuse across capillary walls into the brain, extracellular fluid, and CSF. Larger molecules exchange more slowly and have a larger concentration gradient from serum to CSF than smaller molecules. Some relative concentrations of CSF to serum are IgM 0.0003, IgG 0.002, and albumin $0.005)$ [75]. Brain-derived proteins fall into three categories: 1) Proteins derived from neurons and glial cells like tau, S-100, and neuron-specific enolase, which are released into the ventricular and cisternal CSF, where they have concentrations of 10-, 18-, and 1-fold higher than found in serum, respectively. 2) Proteins derived primarily from the leptomeninges surrounding the brain and spinal cord that are released into the CSF, like beta trace protein (prostaglandin-D-synthase) and cystatin $\mathrm{C}$, which are found at levels 30 and 5 times higher in CSF than serum, respectively; and 3) brain-derived proteins that also have a blood-derived fraction in the CSF, like transthyretin, angiotensin-converting enzyme, and s-ICAM, which typically have lower relative concentrations in the CSF compared to serum (0.05, 0.01, and 0.005 , respectively). In neurological diseases with blood-CSF barrier impairment, all blood proteins are elevated in the CSF.

We collected CSF from 13 patients with MS and 13 control patients, all scheduled to undergo lumbar puncture for medical reasons. A diagnosis of MS was confirmed based on the criteria of McDonald [74]. The control CSF samples were from subjects with a variety of conditions; only subjects with a negative CSF diagnosis were chosen for this study. CSF was processed in a method similar to that described above; however, about 100 times more CSF volume $(0.5 \mathrm{~mL})$ was used than serum in order to load the same amount of protein $(20 \mu \mathrm{g})$ into the liquid chromatography-mass spectrometry instrument. 
Table 1

Summary Statistics for Proteomic profiling of Serum and CSF

\begin{tabular}{|c|c|c|c|c|}
\hline & \multicolumn{2}{|c|}{ Serum } & \multicolumn{2}{|c|}{ CSF } \\
\hline & Control & MS & Control & MS \\
\hline Samples & 20 & 20 & 13 & 13 \\
\hline Components & \multicolumn{2}{|c|}{7308} & \multicolumn{2}{|c|}{4162} \\
\hline Median CV & 27 & 28 & 40 & 35 \\
\hline \multicolumn{5}{|c|}{ Number of significant changes } \\
\hline$P<0.001$ & \multicolumn{2}{|c|}{6} & \multicolumn{2}{|c|}{13} \\
\hline$P<0.01$ & \multicolumn{2}{|c|}{63} & \multicolumn{2}{|c|}{120} \\
\hline$P<0.05$ & \multicolumn{2}{|c|}{313} & \multicolumn{2}{|c|}{400} \\
\hline
\end{tabular}

Proteomic differences between MS and control subjects were found in both the CSF and serum. Summary statistics on the proteomic results are provided in Table 1. The serum samples tracked 1.8 times more molecular components than the CSF samples (7000 vs 4000 components). The median $\mathrm{CVs}$ were lower for the serum samples (28\% for MS and $27 \%$ for controls) than the CSF samples (35\% for MS and $40 \%$ for the controls). The larger CVs for the CSF are likely to reflect greater variation in sample collection and greater variation in the clinical status of the control CSF group. The number of significant changes for the control vs MS group comparisons are presented for each sample type and ranked by p-value. There are multiple comparisons in this study, and more significance should be attached to differences with lower p-values. There were about twice as many differences between the MS and control groups at $\mathrm{p}$-value levels $<0.01$ for CSF than serum. This difference is most significant given both the smaller number of samples and the smaller number of components tracked for CSF. The result strongly supports the hypothesis that CSF is a better source than serum for initial identification of potential biomarkers for MS.

Differential profiling for CSF is presented as a heat map in Fig. 2. The MS and control groups can be readily distinguished by the pattern of expression. The plot shows the relative intensity for components (rows) with p-values $<0.05$ for every subject (columns). Results are sorted by effect size, which is the mean difference between the two groups, divided by the weighted standard deviation of the groups. In addition to betweengroup differences, individual profiles (columns) can be identified in both the MS and control groups, suggesting biological heterogeneity among the subjects within the groups.

As expected, many proteins are found in abundance in serum, and some of these are differentially expressed in MS subjects. Identified proteins come from a variety of biological processes, including inflammation, the complement cascade, lipoproteins and acute phase

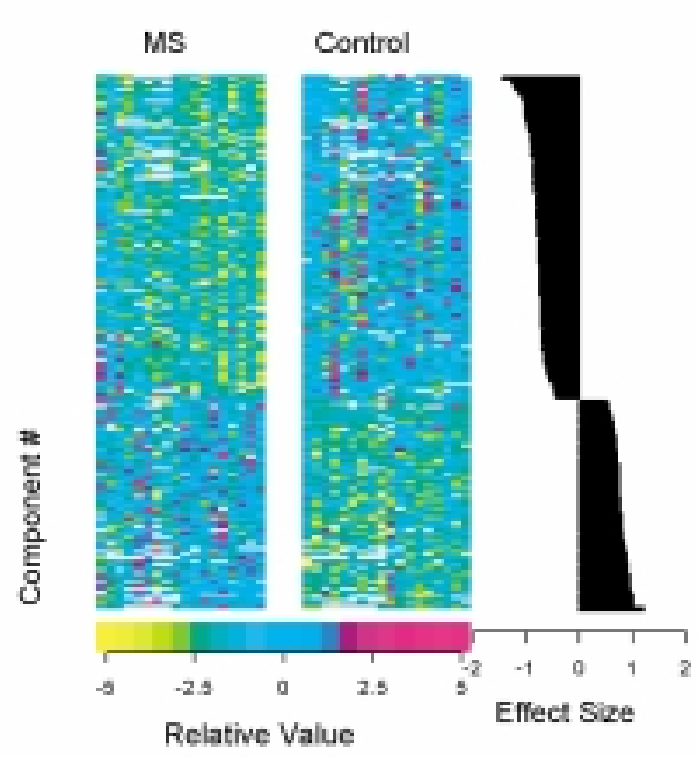

Fig. 2. Proteome Difference Map for CSF comparison. Differences with a p-value $<0.5$ are shown for the MS vs. Control comparison. Each column represents one subject and each row represents one molecular component. Relative value is a normalized z score (Xi$\mathrm{Xave} / \mathrm{SD}$ ), where $\mathrm{Xi}$ is the intensity for sample $\mathrm{i}$, Xave is the average intensity, and SD is the weighted standard deviation. Values are sorted by effect size, the mean difference between the two groups, divided by the weighted standard deviation of the groups. Here, a positive effect size indicates higher means for the MS group.

reactants. This last group of molecules is of particular interest. The acute phase response is a physiologic reaction occurring soon after the onset of infection, trauma, or inflammatory processes. The most prominent change is a dramatic increase of acute phase proteins, which are any of the non-antibody proteins found in increased amounts in serum during this reaction; examples include $\mathrm{C}$-reactive protein, serum amyloid $\mathrm{A}$ protein, fibrinogen and 1-acid glycoprotein. The concentrations of many of the proteins within this collection have been reported to be altered in MS.

At this point, about $15 \%$ of the components in the data set have been identified. Some of the specific differences between MS and control subjects are presented in Fig. 3. The x-axis plots the effect size. Most of the proteins present in Fig. 3 are tracked with more than one peptide, as indicated in the parentheses. This consistency increases our confidence in individual protein results. An example of this is seen in the consistent results for leucine-rich alpha-2-glycoprotein in serum, with three different peptides shown. Several of the components presented, such as A2GP1 and haptoglobin, are acute phase reactants that could be as- 


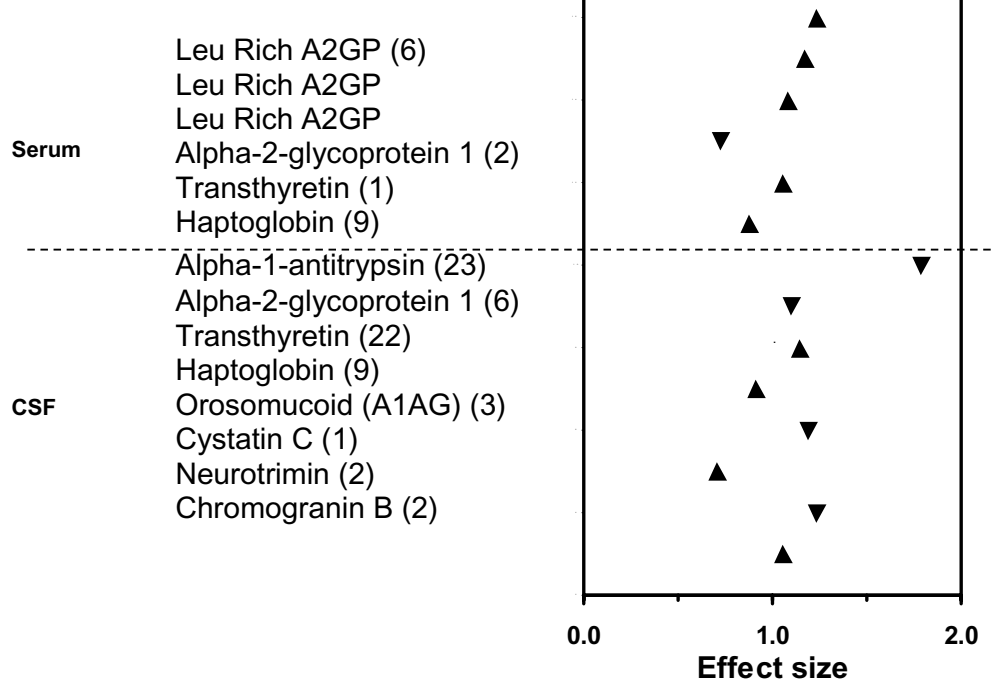

Fig. 3. Proteomic differences between MS subjects and controls. The effect size, the mean difference between the two groups, divided by the weighted standard deviation of the groups, is shown for a subset of identified components with $p<0.05$. The direction of the triangle symbols are $(\boldsymbol{\Delta})$ higher in controls, $(\boldsymbol{\nabla})$ lower in controls. Numbers in parentheses are the consistent components for that protein with $p<0.05$.

sociated with inflammatory conditions. Some other acute phase reactants, like alpha-1 antitrypsin, were not significantly elevated in serum.

The effect sizes shown in Fig. 3 are quite substantial, with several greater than one. For reference, in another neurological disorder, Tau and $\mathrm{A} \beta$ peptide have an effect size of about one in Alzheimer's disease vs either age-matched healthy controls or age-matched dementia controls. Our calculation is based on data from Mulder et al and references therein [76]. We have also completed a proteomic analysis of serum samples from rheumatoid arthritis subjects with active disease (tender/swollen joints, elevated erythrocyte sedimentation rate, etc) and controls [65]. A broader constellation of markers consistent with a chronic inflammatory condition was observed in the rheumatoid arthritis study. Many have effect sizes greater than one. The relapsingremitting MS subjects in the current study do not necessarily have active clinical events.

Many of the proteins identified in serum are also detected in the CSF. These include alpha-1-antitrypsin, alpha-1-acid glycoprotein, haptoglobin, transferrin, and transthyretin. Interestingly, studies that have associated these molecules with MS have been reported: Proteases and their inhibitors have been implicated in the pathogenesis of MS. Levels of the protease inhibitor, alpha-1-antitrypsin, in the CSF have been associated with disturbances in the BBB [77]. The acute phase protein, alpha-1-acid glycoprotein (orosomucoid) levels are altered in the CSF of patients with
MS [78]. Although the function of alpha-1-acid glycoprotein is unknown, it has protective effects in several in vivo models of inflammation [79]. Haptoglobin irreversibly binds free hemoglobin to facilitate its removal and increased synthesis of haptoglobin is observed in conditions of extensive tissue damage and necrosis. Its altered levels have been observed in inflammatory neurological diseases [80]. Studies indicate that free radicals participate in the pathogenesis of MS, and iron has been implicated as the catalyst leading to their formation. Thus, proteins involved with iron metabolism, such as transferrin, have been proposed as putative biomarkers for demyelination [81]. Finally, the level of transthyretin, a choroid plexusspecific transport protein, has been reported to be elevated, decreased and unaffected in the CSF of patients with MS [82]. Our finding that it is elevated relative to the non-MS group underscores our belief that many of these potential markers warrant re-evaluation with new and more sensitive assays.

We also identified several proteins produced in the central nervous system. These include beta trace protein (prostaglandin-D-synthase) and cystatin C (gamma trace), which are produced by the cells of the cerebral cortex and choroid plexus, and transthyretin, which is synthesized both by the choroid plexus and by the liver. Acute phase proteins e.g. alpha-1-antitrypsin, alpha-1-acid glycoprotein haptoglobin, and transferrin show stronger and more consistent effects in the CSF than serum. Often more consistent peptides per protein 

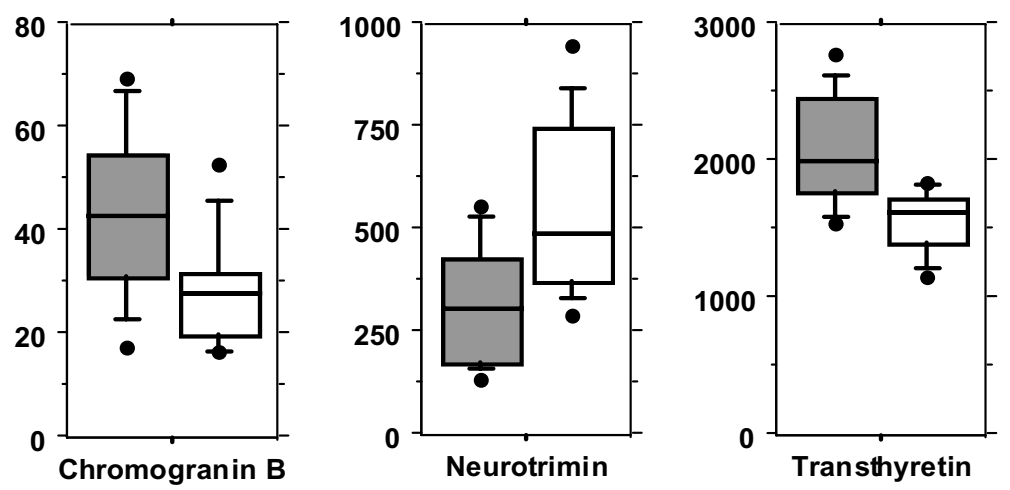

Fig. 4. Distributions for CSF proteomic differences between MS subjects and controls. Results are shown for representative peptides for chromogranin B (effect size $=1.1, p=0.15,2$ identified components), neurotrimin (effect size $=1.2, p=0.0047,2$ identified components), and transthyretin (effect size $=1.7, p=0.00042,22$ identified components). Box and whisker plots: bottom line $=10^{\mathrm{h}} \%$, bottom box $=25^{\text {th }}$ $\%$, center line $=$ median, top box $=75^{\text {th }} \%$, top line $=90^{\text {th }} \%$, circles are individual points outside the range. Grey $=$ MS, White $=$ control.

are different in the CSF. Among proteins produced in the brain, gamma trace (cystatin $C$ ), neurotrimin, chromogranin B, and transthyretin are significantly different between the MS and control groups. Distributions for representative peptides from three of these proteins identified in CSF are presented as box and whisker plots in Fig. 4. All have effect sizes greater than one, which corresponds to the boxes of the MS and control groups being almost separated. The displayed proteins are of neurological interest. Chromogranin B (secretonin 1), a neuroendocrine secretory granule protein produced in the brain and pancreas, is more abundant in the CSF of MS subjects. The single study we found, reported that the CSF levels of both chromogranin A and B are similar among MS and controls groups [83], emphasizing again that further investigation is required. Neurotrimin, a neural cell adhesion molecule produced in the brain as well as muscle, liver, and other tissues, is found at lower levels in the CSF in MS subjects. Interestingly, this protein has been implicated, in part, in regulating the development of neuronal projections and axonal fasciculation $[84,85]$. Transthyretin (prealbumin), which probably transports thyroxine from the bloodstream to the brain, is elevated in the CSF of MS subjects. All three proteins have potential connections to MS disease physiology and require more extensive evaluation.

\section{Summary and conclusions}

Exploration of the etiology and pathogenesis of MS has yielded a handful of useful biomarkers. Markers of both autoimmune and neurodegeneration processes have been reported. Still, the need for markers for accurate diagnosis, course of severity, likelihood of onset, and determinants of etiology and pathogenesis are desired. The existence of informative single MS biomarkers in such a complex system is not likely. A pattern of many single molecules that are not useful individually may hold the key. Our strategy of comprehensive phenotyping based on discovery rather than hypothesis holds promise for elucidation of such markers. In addition to these potential benefits, this strategy offers the researcher an extensive library of new candidate molecules for support of current theories or development of new ideas in the pathophysiology of the disease. Finally, it must be considered that the molecular fingerprint of a patient with MS is likely to evolve over time relative to the underlying pathology. Recognition and characterization of this alteration will also become a rich source of information regarding the disease process and may yield predictors of various disease parameters in multiple sclerosis.

\section{Acknowledgements}

KCO is a National Multiple Sclerosis Society Career Transition Award recipient (TA3000A2). DAH is an $\mathrm{NIH}$, Javits Investigator Award recipient. This work was funded, in part, through a grant from The Autoimmune Prevention Center awarded to DAH, ABK, and KCO and by NIH NINDS Grant R43 NS046107 to ABK and by the NIH grants: UO1DK6192601, RO1NS2424710, PO1AI39671, and PO1NS38037; and grants from the National Multiple Sclerosis Society: RG2172C9 and RG3308A10 to DAH. We thank 
Jeffrey Satkofsky and Hua Lin for contributions to the mass spectrometry work, and Praveen Kumar and Markus Anderle for contributions in the areas of data processing and statistics and Paul Guttry for editorial assistance.

\section{References}

[1] B. Bielekova and R. Martin, Development of biomarkers in multiple sclerosis, Brain 127(Pt 7) (2004), 1463-1478.

[2] A. Miller, L. Glass-Marmor, M. Abraham, I. Grossman, S. Shapiro and Y. Galboiz, Bio-markers of disease activity and response to therapy in multiple sclerosis, Clin Neurol Neurosurg 106(3) (2004), 249-254.

[3] C.E. Teunissen, C. Dijkstra and C. Polman, Biological markers in CSF and blood for axonal degeneration in multiple sclerosis, Lancet Neurol 4(1) (2005), 32-41.

[4] D.A. Hafler, Multiple sclerosis, J Clin Invest 113(6) (2004), 788-794.

[5] J.H. Noseworthy, C. Lucchinetti, M. Rodriguez and B.G. Weinshenker, Multiple sclerosis, $N$ Engl J Med 343(13) (2000), 938-952.

[6] K. Whetten-Goldstein, F.A. Sloan, L.B. Goldstein and E.D. Kulas, A comprehensive assessment of the cost of multiple sclerosis in the United States, Mult Scler 4(5) (1998), 419-425.

[7] C. Bjartmar and B.D. Trapp, Axonal and neuronal degeneration in multiple sclerosis: mechanisms and functional consequences, Curr Opin Neurol 14(3) (2001), 271-278.

[8] H. Lassmann, Neuropathology in multiple sclerosis: new concepts, Mult Scler 4(3) (1998), 93-98.

[9] K.C. O'Connor, A. Bar-Or and D.A. Hafler, The neuroimmunology of multiple sclerosis: possible roles of $\mathrm{T}$ and $\mathrm{B}$ lymphocytes in immunopathogenesis, J Clin Immunol 21(2) (2001), 81-92.

[10] D.A. Hafler, J.M. Slavik, D.A. Anderson, K.C. O'Connor, P. De Jager and C.M. Baecher-Allan, Multiple Sclerosis, Immunol Rev 204 (2005), 208-231.

[11] A. Bar-Or, E.M. Oliveira, D.E. Anderson and D.A. Hafler, Molecular pathogenesis of multiple sclerosis, J Neuroimmunol 100(1-2) (1999), 252-259.

[12] D.A. Hafler and H.L. Weiner, Immunologic mechanisms and therapy in multiple sclerosis, Immunol Rev 144 (1995), 75107.

[13] J.W. Peterson and B.D. Trapp, Neuropathobiology of multiple sclerosis, Neurol Clin 23(1) (2005), 107-129, vi-vii.

[14] A. Chaudhuri and P.O. Behan, Multiple sclerosis is not an autoimmune disease, Arch Neurol 61(10) (2004), 1610-1612.

[15] T. Chitnis, J. Imatola and S.J. Khoury, Therapeutic Strategies To Prevent Neurodegeneration And Promote Regeneration In Multiple Sclerosis, Current Drug Targets - Immune, Endocrine \& Metabolic Disorders (2005), in press.

[16] C. Lucchinetti, W. Bruck, J. Parisi, B. Scheithauer, M. Rodriguez and H. Lassmann, Heterogeneity of multiple sclerosis lesions: implications for the pathogenesis of demyelination, Ann Neurol 47(6) (2000), 707-717.

[17] Biomarkers and surrogate endpoints: preferred definitions and conceptual framework, Clin Pharmacol Ther 69(3) (2001), 89-95.

[18] A.I. Danilov, M. Andersson, N. Bavand, N.P. Wiklund, T. Olsson and L. Brundin, Nitric oxide metabolite determinations reveal continuous inflammation in multiple sclerosis, $J$ Neuroimmunol 136(1-2) (2003), 112-118.
[19] A. Vincent, Unravelling the pathogenesis of myasthenia gravis, Nat Rev Immunol 2(10) (2002), 797-804.

[20] V.A. Lennon, D.M. Wingerchuk, T.J. Kryzer, S.J. Pittock, C.F. Lucchinetti, K. Fujihara, I. Nakashima and B.G. Weinshenker, A serum autoantibody marker of neuromyelitis optica: distinction from multiple sclerosis, Lancet 364(9451) (2004), 2106-2112.

[21] O. Lily, J. Palace and A. Vincent, Serum autoantibodies to cell surface determinants in multiple sclerosis: a flow cytometric study, Brain 127(Pt 2) (2004), 269-279.

[22] A. Lowenthal, M. Van Sande and D. Karcher, The differential diagnosis of neurological diseases by fractionating electrophoretically the CSF G-globulins, J Neurochem 235 (1960), 229-233.

[23] E.A. Kabat, D.A. Freedman, J.P. Murray and V. Knaub, A study of the crystalline albumin, gamma globulin and the total protein in the cerebrospinal fluid of one hundred cases of multiple sclerosis and other diseases, Am J Med Sci 219 (1950), 55-64.

[24] H.S. Panitch, D.A. Hafler and K.P. Johnson, Antibodies to myelin basic protein in cerebrospinal fluid of patients with multiple sclerosis, in: Progress in Multiple Sclerosis Research, H.J. Bauer, S. Poser and G. Ritter, eds, Springer-Verlag: Berlin, 1980, 98-105.

[25] K.G. Warren and I. Catz, A correlation between cerebrospinal fluid myelin basic protein and anti-myelin basic protein in multiple sclerosis patients, Ann Neurol 21(2) (1987), 183-189.

[26] K.G. Warren and I. Catz, An extensive search for autoantibodies to myelin basic protein in cerebrospinal fluid of nonmultiple-sclerosis patients: implications for the pathogenesis of multiple sclerosis, Eur Neurol 42(2) (1999), 95-104.

[27] J.R. Moller, D. Johnson, R.O. Brady, W.W. Tourtellotte and R.H. Quarles, Antibodies to myelin-associated glycoprotein (MAG) in the cerebrospinal fluid of multiple sclerosis patients, J Neuroimmunol 22(1) (1989), 55-61.

[28] K. Banki, E. Colombo, F. Sia, D. Halladay, D.H. Mattson, A.H. Tatum, P.T. Massa, P.E. Phillips and A. Perl, Oligodendrocytespecific expression and autoantigenicity of transaldolase in multiple sclerosis, J Exp Med 180(5) (1994), 1649-1663.

[29] B.G. Xiao, C. Linington and H. Link, Antibodies to myelinoligodendrocyte glycoprotein in cerebrospinal fluid from patients with multiple sclerosis and controls, Journal of $\mathrm{Neu}$ roimmunology 31(2) (1991), 91-96.

[30] J.M. Bronstein, R.L. Lallone, R.S. Seitz, G.W. Ellison and L.W. Myers, A humoral response to oligodendrocyte-specific protein in MS: a potential molecular mimic, Neurology 53(1) (1999), 154-161.

[31] L.M. Villar, J. Masjuan, P. Gonzalez-Porque, J. Plaza, M.C. Sadaba, E. Roldan, A. Bootello and J.C. Alvarez-Cermeno, Intrathecal IgM synthesis is a prognostic factor in multiple sclerosis, Ann Neurol 53(2) (2003), 222-226.

[32] L.M. Villar, J. Masjuan, P. Gonzalez-Porque, J. Plaza, M.C. Sadaba, E. Roldan, A. Bootello and J.C. Alvarez-Cermeno, Intrathecal $\operatorname{IgM}$ synthesis predicts the onset of new relapses and a worse disease course in MS, Neurology 59(4) (2002), $555-559$.

[33] L.M. Villar, J. Masjuan, P. Gonzalez-Porque, J. Plaza, M.C. Sadaba, E. Roldan, A. Bootello and J.C. Alvarez-Cermeno, Intrathecal IgM synthesis in neurologic diseases: relationship with disability in MS, Neurology 58(5) (2002), 824-826.

[34] L.M. Villar, M.C. Sadaba, E. Roldan, J. Masjuan, P. GonzalezPorque, N. Villarrubia, M. Espino, J.A. Garcia-Trujillo, A. Bootello and J.C. Alvarez-Cermeno, Intrathecal synthesis of 
oligoclonal IgM against myelin lipids predicts an aggressive disease course in MS, J Clin Invest 115(1) (2005), 187-194.

[35] M. Reindl, C. Linington, U. Brehm, R. Egg, E. Dilitz, F. Deisenhammer, W. Poewe and T. Berger, Antibodies against the myelin oligodendrocyte glycoprotein and the myelin basic protein in multiple sclerosis and other neurological diseases: a comparative study, Brain 122(Pt 11) (1999), 2047-2056.

[36] J.W. Terryberry, G. Thor and J.B. Peter, Autoantibodies in neurodegenerative diseases: antigen-specific frequencies and intrathecal analysis, Neurobiol Aging 19(3) (1998), 205-216.

[37] K.A. Brokstad, M. Page, H. Nyland and L.R. Haaheim, Autoantibodies to myelin basic protein are not present in the serum and CSF of MS patients, Acta Neurol Scand 89(6) (1994), 407-411.

[38] T. Olsson, S. Baig, B. Hojeberg and H. Link, Antimyelin basic protein and antimyelin antibody-producing cells in multiple sclerosis, Ann Neurol 27(2) (1990), 132-136.

[39] E. Colombo, K. Banki, A.H. Tatum, J. Daucher, P. Ferrante, R.S. Murray, P.E. Phillips and A. Perl, Comparative analysis of antibody and cell-mediated autoimmunity to transaldolase and myelin basic protein in patients with multiple sclerosis, $J$ Clin Invest 99(6) (1997), 1238-1250.

[40] R.B. Lindert, C.G. Haase, U. Brehm, C. Linington, H. Wekerle and R. Hohlfeld, Multiple sclerosis: B- and T-cell responses to the extracellular domain of the myelin oligodendrocyte glycoprotein, Brain 122(Pt 11) (1999), 2089-2100.

[41] J.B. Sun, T. Olsson, W.Z. Wang, B.G. Xiao, V. Kostulas, S. Fredrikson, H.P. Ekre and H. Link, Autoreactive T and B cells responding to myelin proteolipid protein in multiple sclerosis and controls, Eur J Immunol 21(6) (1991), 1461-1468.

[42] S. Schmidt, Candidate autoantigens in multiple sclerosis, Mult Scler 5(3) (1999), 147-160.

[43] S. Barned, A.D. Goodman and D.H. Mattson, Frequency of anti-nuclear antibodies in multiple sclerosis, Neurology $\mathbf{4 5}(2)$ (1995), 384-385.

[44] C.B. Colaco, G.K. Scadding and S. Lockhart, Anti-cardiolipin antibodies in neurological disorders: cross-reaction with antisingle stranded DNA activity, Clin Exp Immunol 68(2) (1987), 313-319.

[45] V. Roussel, F. Yi, M.O. Jauberteau, C. Couderq, C. Lacombe, V. Michelet, R. Gil, P. Couratier, J.M. Vallat and J.L. Preud'homme, Prevalence and clinical significance of antiphospholipid antibodies in multiple sclerosis: a study of 89 patients, J Autoimmun 14(3) (2000), 259-265.

[46] M. Spadaro, M.A. Amendolea, M.G. Mazzucconi, R. Fantozzi, R. Di Lello, P. Zangari and G. Masala, Autoimmunity in multiple sclerosis: study of a wide spectrum of autoantibodies, Mult Scler 5(2) (1999), 121-125.

[47] K.C. O'Connor, T. Chitnis, D.E. Griffin, S. Piyasirisilp, A. Bar-Or, S. Khoury, K.W. Wucherpfennig and D.A. Hafler, Myelin basic protein-reactive autoantibodies in the serum and cerebrospinal fluid of multiple sclerosis patients are characterized by low-affinity interactions, J Neuroimmunol 136(1-2) (2003), 140-148.

[48] K.C. O'Connor, H. Appel, M.E. Call, J.A. Chan, N.H. Moore, D.A. Hafler and K.W. Wucherpfennig, Isolation of autoantibodies recognizing MOG from CNS tissue of patients with MS, Submitted (2005).

[49] S. Piyasirisilp, T. Hemachudha and D.E. Griffin, B-cell responses to myelin basic protein and its epitopes in autoimmune encephalomyelitis induced by Semple rabies vaccine, $J$ Neuroimmunol 98(2) (1999), 96-104.
[50] J.W. Prineas and R.G. Wright, Macrophages, lymphocytes, and plasma cells in the perivascular compartment in chronic multiple sclerosis, Lab Invest 38(4) (1978), 409-421.

[51] Y. Qin, P. Duquette, Y. Zhang, P. Talbot, R. Poole and J. Antel, Clonal expansion and somatic hypermutation of $\mathrm{V}(\mathrm{H})$ genes of B cells from cerebrospinal fluid in multiple sclerosis, $\mathrm{J}$ Clin Invest 102(5) (1998), 1045-1050.

[52] R.A. Williamson, M.P. Burgoon, G.P. Owens, O. Ghausi, E. Leclerc, L. Firme, S. Carlson, J. Corboy, P.W. Parren, P.P. Sanna, D.H. Gilden and D.R. Burton, Anti-DNA antibodies are a major component of the intrathecal B cell response in multiple sclerosis, Proc Natl Acad Sci USA 98(4) (2001), 1793-1798.

[53] T. Smith-Jensen, M.P. Burgoon, J. Anthony, H. Kraus, D.H. Gilden and G.P. Owens, Comparison of immunoglobulin G heavy-chain sequences in MS and SSPE brains reveals an antigen-driven response, Neurology 54(6) (2000), 1227-1232.

[54] M. Colombo, M. Dono, P. Gazzola, S. Roncella, A. Valetto, N. Chiorazzi, G.L. Mancardi and M. Ferrarini, Accumulation of clonally related B lymphocytes in the cerebrospinal fluid of multiple sclerosis patients, J Immunol 164(5) (2000), 27822789.

[55] S.E. Baranzini, M.C. Jeong, C. Butunoi, R.S. Murray, C.C. Bernard and J.R. Oksenberg, B cell repertoire diversity and clonal expansion in multiple sclerosis brain lesions, J Immunol 163(9) (1999), 5133-5144.

[56] C.P. Genain, B. Cannella, S.L. Hauser and C.S. Raine, Identification of autoantibodies associated with myelin damage in multiple sclerosis, Nat Med 5(2) (1999), 170-175.

[57] K.G. Warren and I. Catz, Autoantibodies to myelin basic protein within multiple sclerosis central nervous system tissue, $J$ Neurol Sci 115(2) (1993), 169-176.

[58] M.H. Barnett and J.W. Prineas, Relapsing and remitting multiple sclerosis: pathology of the newly forming lesion, Ann Neurol 55(4) (2004), 458-468.

[59] A. Petzold, M.J. Eikelenboom, D. Gveric, G. Keir, M. Chapman, R.H. Lazeron, M.L. Cuzner, C.H. Polman, B.M. Uitdehaag, E.J. Thompson and G. Giovannoni, Markers for different glial cell responses in multiple sclerosis: clinical and pathological correlations, Brain 125(Pt 7) (2002), 1462-1473.

[60] A. Greco, L. Minghetti, G. Sette, C. Fieschi and G. Levi, Cerebrospinal fluid isoprostane shows oxidative stress in patients with multiple sclerosis, Neurology 53(8) (1999), 1876-1879.

[61] A. Greco, L. Minghetti, M. Puopolo, S. Cannoni, S. Romano, C. Pozzilli and G. Levi, Cerebrospinal fluid isoprostanes are not related to inflammatory activity in relapsing-remitting multiple sclerosis, J Neurol Sci 224(1-2) (2004), 23-27.

[62] M. Zaffaroni, Biological indicators of the neurodegenerative phase of multiple sclerosis, Neurol Sci 24(Suppl 5) (2003), S279-S282.

[63] M.J. Eikelenboom, A. Petzold, R.H. Lazeron, E. Silber, M. Sharief, E.J. Thompson, F. Barkhof, G. Giovannoni, C.H. Polman and B.M. Uitdehaag, Multiple sclerosis: Neurofilament light chain antibodies are correlated to cerebral atrophy, $\mathrm{Neu}$ rology 60(2) (2003), 219-223.

[64] A.B. Kantor, Comprehensive phenotyping and biological marker discovery, Dis Markers 18(2) (2002), 91-97.

[65] A.B. Kantor, W. Wang, H. Lin, H. Govindarajan, M. Anderle, A. Perrone and C. Becker, Biomarker discovery by comprehensive phenotyping for autoimmune diseases, Clin Immunol 111(2) (2004), 186-195.

[66] A.B. Kantor, S.E. Alters, K. Cheal and L.J. Dietz, Immune systems biology: immunoprofiling of cells and molecules, Biotechniques 36(3) (2004), 520-524. 
[67] W. Wang, H. Zhou, H. Lin, S. Roy, T.A. Shaler, L.R. Hill, S. Norton, P. Kumar, M. Anderle and C.H. Becker, Quantification of proteins and metabolites by mass spectrometry without isotopic labeling or spiked standards, Anal Chem 75(18) (2003), 4818-4826.

[68] S. Roy, M. Anderle, H. L., and C. Becker, Differential expression profiling of serum proteins and metabolites for biomarker discovery, Int J. of Mass Spectrometry 238 (2004), 163-171.

[69] S.M. Roy and C.H. Becker, Quantification of Proteins and Metabolites by Mass Spectrometry Without Isotopic Labeling, in: Methods in Molecular Biology: An Overview of Recent Developments in Quantitative Proteomics using Mass Spectrometry, S. Sechi, ed., 2006, Humana Press.

[70] R. Aebersold and M. Mann, Mass spectrometry-based proteomics, Nature 422(6928) (2003), 198-207.

[71] C.A. Hastings, S.M. Norton and S. Roy, New algorithms for processing and peak detection in liquid chromatography/mass spectrometry data, Rapid Commun Mass Spectrom 16(5) (2002), 462-467.

[72] C. Becker, C.A. Hastings and S. Norton, Mass spectrometric quantification of chemcial mixture components, in Patent No 6,835,927. 2002: USA

[73] A.J. Link, J. Eng, D.M. Schieltz, E. Carmack, G.J. Mize, D.R. Morris, B.M. Garvik and J.R. Yates, 3rd, Direct analysis of protein complexes using mass spectrometry, Nat Biotechnol 17(7) (1999), 676-682.

[74] W.I. McDonald, A. Compston, G. Edan, D. Goodkin, H.P. Hartung, F.D. Lublin, H.F. McFarland, D.W. Paty, C.H. Polman, S.C. Reingold, M. Sandberg-Wollheim, W. Sibley, A. Thompson, S. van den Noort, B.Y. Weinshenker and J.S. Wolinsky, Recommended diagnostic criteria for multiple sclerosis: guidelines from the International Panel on the diagnosis of multiple sclerosis, Ann Neurol 50(1) (2001), 121-127.

[75] H. Reiber and J.B. Peter, Cerebrospinal fluid analysis: diseaserelated data patterns and evaluation programs, $J$ Neurol Sci 184(2) (2001), 101-22.

[76] C. Mulder, P. Scheltens, J.J. Visser, G.J. van Kamp and R.B. Schutgens, Genetic and biochemical markers for Alzheimer's disease: recent developments, Ann Clin Biochem 37(Pt 5) (2000), 593-607.

[77] G.S. Pearl and R.E. Mullins, Alpha 1-antitrypsin in cerebrospinal fluid of patients with neurologic diseases, $\mathrm{Arch} \mathrm{Neu-}$ rol 42(8) (1985), 775-777.

[78] P. Adam, O. Sobek, L. Taborsky, T. Hildebrand, O. Tutterova and P. Zacek, CSF and serum orosomucoid (alpha-1-acid glycoprotein) in patients with multiple sclerosis: a comparison among particular subgroups of MS patients, Clin Chim Acta 334(1-2) (2003), 107-110.

[79] T. Hochepied, F.G. Berger, H. Baumann and C. Libert, Alpha(1)-acid glycoprotein: an acute phase protein with inflammatory and immunomodulating properties, Cytokine Growth Factor Rev 14(1) (2003), 25-34.

[80] N.J. Gutowski, J.M. Pinkham, D. Akanmu, S. Chirico and R.P. Murphy, Free radicals in inflammatory neurological disease: increased lipid peroxidation and haptoglobin levels in Guillain Barre syndrome, Ir J Med Sci 167(1) (1998), 43-46.

[81] S.M. Levine and A. Chakrabarty, The role of iron in the pathogenesis of experimental allergic encephalomyelitis and multiple sclerosis, Ann N Y Acad Sci 1012 (2004), 252-266.

[82] P.D. Larsen and L.J. DeLallo, Cerebrospinal fluid transthyretin in multiple sclerosis, Neurology 37(7) (1987), 1262-1263

[83] U. Eder, B. Leitner, R. Kirchmair, P. Pohl, K.A. Jobst, A.D. Smith, J. Mally, A. Benzer, P. Riederer, H. Reichmann, A. Saria and $\mathrm{H}$. Winkler, Levels and proteolytic processing of chromogranin A and B and secretogranin II in cerebrospinal fluid in neurological diseases, J Neural Transm 105(1) (1998), 39-51.

[84] S. Chen, O. Gil, Y.Q. Ren, G. Zanazzi, J.L. Salzer and D.E. Hillman, Neurotrimin expression during cerebellar development suggests roles in axon fasciculation and synaptogenesis, J Neurocytol 30(11) (2001), 927-937.

[85] O.D. Gil, G. Zanazzi, A.F. Struyk and J.L. Salzer, Neurotrimin mediates bifunctional effects on neurite outgrowth via homophilic and heterophilic interactions, J Neurosci 18(22) (1998), 9312-9325. 


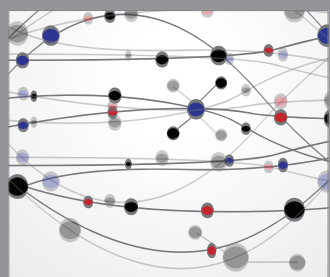

The Scientific World Journal
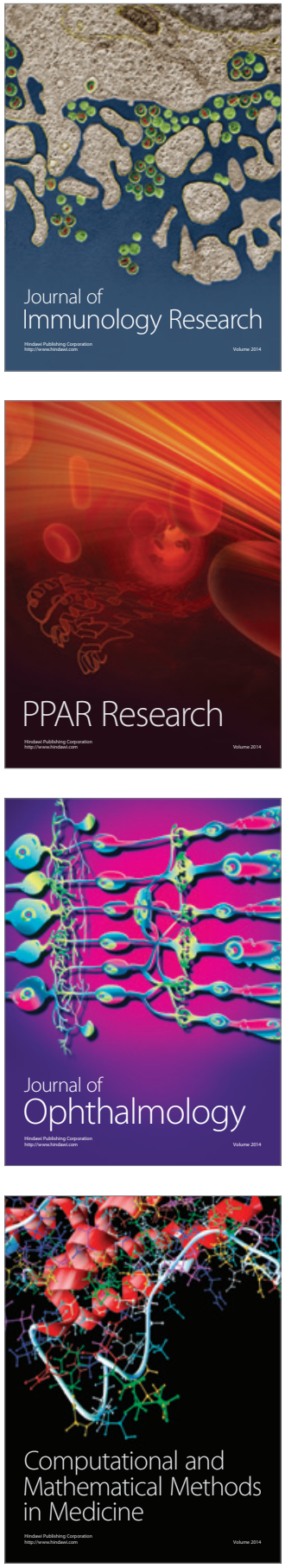

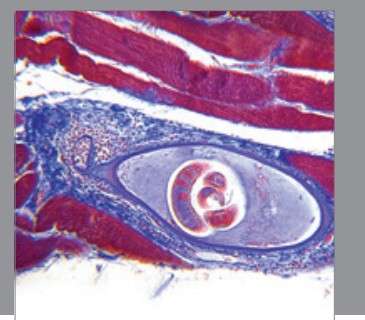

Gastroenterology

Research and Practice
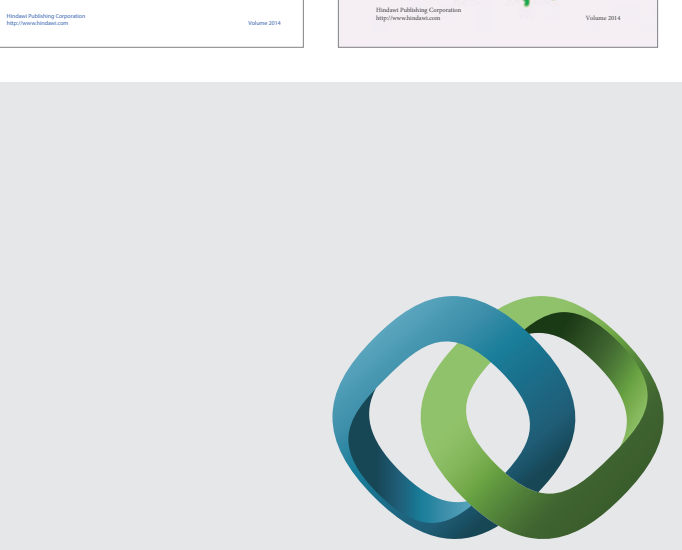

\section{Hindawi}

Submit your manuscripts at

http://www.hindawi.com
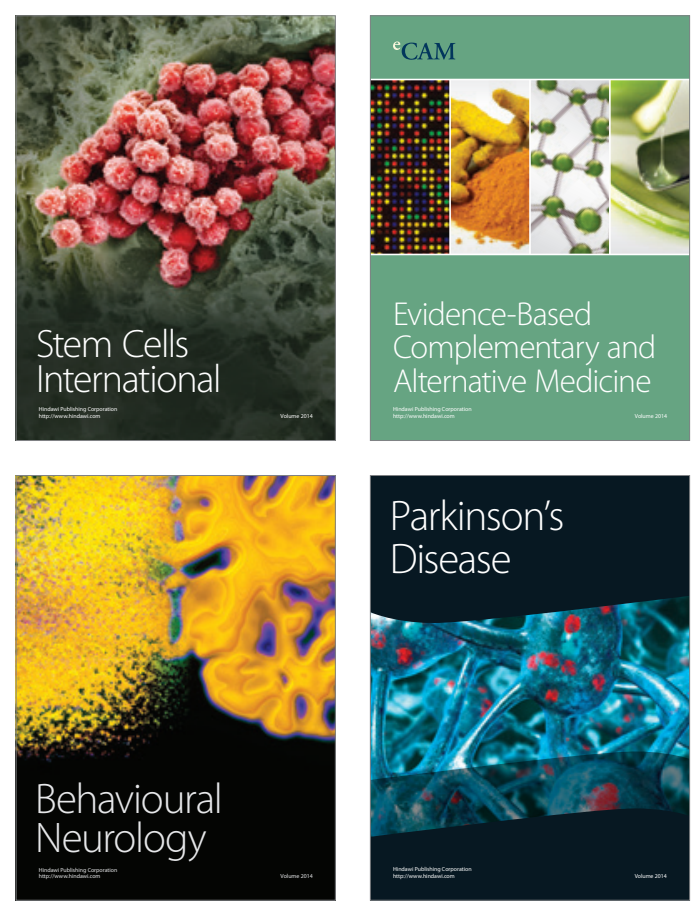

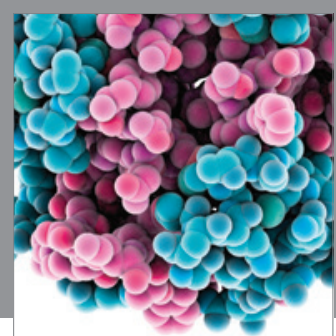

Journal of
Diabetes Research

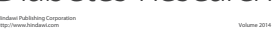

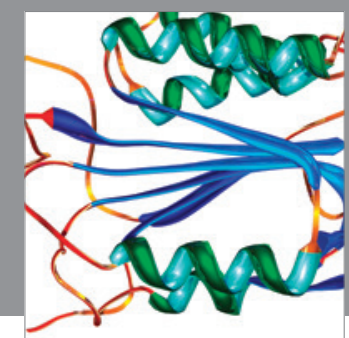

Disease Markers
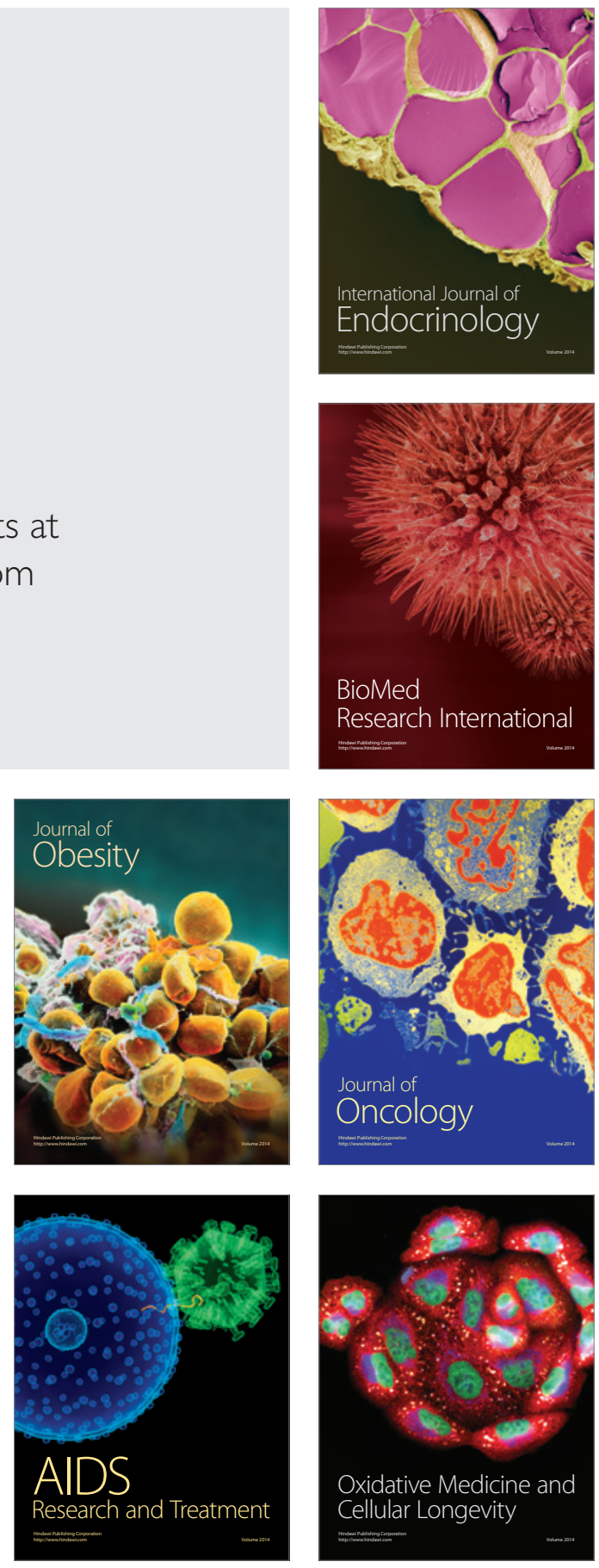